\title{
Certain Properties Of A Subclass Of Harmonic Functions
}

\author{
Elif Yaşar* and Sibel Yalçın \\ Department of Mathematics, Faculty of Arts and Science, Uludag University, 16059, Bursa, Turkey
}

Received: 4 Jan. 2013, Revised: 6 May. 2013, Accepted: 8 May. 2013

Published online: 1 Sep. 2013

\begin{abstract}
In the present paper, we investigate some basic properties of a subclass of harmonic functions defined by multiplier transformations. Such as, coefficient inequalities, distortion bounds and extreme points.
\end{abstract}

Keywords: Harmonic, univalent, modified Salagean operator, multiplier transformation.

\section{Introduction}

Let $H$ denote the family of continuous complex valued harmonic functions which are harmonic in the open unit disk $U=\{z:|z|<1\}$ and let $A$ be the subclass of $H$ consisting of functions which are analytic in $U$. A function harmonic in $U$ may be written as $f=h+\bar{g}$, where $h$ and $g$ are members of $A$. In this case, $f$ is sense-preserving if $\left|h^{\prime}(z)\right|>\left|g^{\prime}(z)\right|$ in $U$. See Clunie and Sheil-Small [4]. To this end, without loss of generality, we may write

$$
h(z)=z+\sum_{k=2}^{\infty} a_{k} z^{k}, \quad g(z)=\sum_{k=1}^{\infty} b_{k} z^{k} .
$$

Let $S H$ denote the family of functions $f=h+\bar{g}$ which are harmonic, univalent, and sense-preserving in $U$ for which $f(0)=f_{z}(0)-1=0$. One shows easily that the sense-preserving property implies that $\left|b_{1}\right|<1$. The subclass $\mathrm{SH}^{0}$ of $\mathrm{SH}$ consists of all functions in $\mathrm{SH}$ which have the additional property $f_{\bar{z}}(0)=0$.

Note that $S H$ reduces to the class $S$ of normalized analytic univalent functions in $U$ if the co-analytic part of $f$ is identically zero.

In 1984 Clunie and Sheil-Small [4] investigated the class $S H$ as well as its geometric subclasses and obtained some coefficient bounds. Since then, there has been several related papers on $S H$ and its subclasses such as Avc1 and Zlotkiewicz [1], Silverman [9], Silverman and Silvia [10], Jahangiri [6] studied the harmonic univalent functions.

For $f \in S$, the differential operator $D^{n}\left(n \in \mathbb{N}_{0}\right)$ of $f$ was introduced by Salagean [8]. For $f=h+\bar{g}$ given by (1),
Jahangiri et al. [7] defined the modified Salagean operator of $f$ as

$$
D^{n} f(z)=D^{n} h(z)+(-1)^{n} \overline{D^{n} g(z)},
$$

where

$$
D^{n} h(z)=z+\sum_{k=2}^{\infty} k^{n} a_{k} z^{k} \text { and } D^{n} g(z)=\sum_{k=1}^{\infty} k^{n} b_{k} z^{k} .
$$

Next, for functions $f \in A$, Cho and Srivastava [2] defined multiplier transformations. For $f=h+\bar{g}$ given by (1), we define the modified multiplier transformation of $f$

$$
I_{\gamma}^{0} f(z)=D^{0} f(z)=h(z)+\overline{g(z)},
$$

$$
\begin{gathered}
I_{\gamma}^{1} f(z)=\frac{\gamma D^{0} f(z)+D^{1} f(z)}{\gamma+1}=\frac{\gamma h(z)+\gamma \overline{g(z)}+z h^{\prime}(z)-\overline{z g^{\prime}(z)}}{\gamma+1}, \quad \gamma \geq 0 \\
I_{\gamma}^{n} f(z)=I_{\gamma}^{1}\left(I_{\gamma}^{n-1} f(z)\right) \cdot\left(n \in \mathbb{N}_{0}\right)
\end{gathered}
$$

If $f$ is given by (1), then from (2) and (3) we see that

$$
I_{\gamma}^{n} f(z)=z+\sum_{k=2}^{\infty}\left(\frac{k+\gamma}{1+\gamma}\right)^{n} a_{k} z^{k}+(-1)^{n} \sum_{k=1}^{\infty}\left(\frac{k-\gamma}{1+\gamma}\right)^{n} \overline{b_{k} z^{k}}
$$

Also if $f$ is given by (1), then we have

$$
\begin{aligned}
I_{\gamma}^{n} f(z) & :=f \tilde{*} \underbrace{\left(\phi_{1}(z)+\overline{\phi_{2}(z)}\right) \tilde{*} \ldots \tilde{*}\left(\phi_{1}(z)+\overline{\phi_{2}(z)}\right)}_{n \text { times }}, \overline{g * \underbrace{\phi_{1}(z) * \ldots * \phi_{1}(z)}_{n \text { times }}+\ldots * \phi_{2}(z)}, \\
& =h \underbrace{\phi_{1}(z)}_{\text {times }}
\end{aligned}
$$

\footnotetext{
* Corresponding author e-mail: elifyasar@uludag.edu.tr
} 
where $*$ denotes the usual Hadamard product or convolution of power series and

$$
\phi_{1}(z)=\frac{(1+\gamma) z-\gamma z^{2}}{(1+\gamma)(1-z)^{2}}, \quad \phi_{2}(z)=\frac{(\gamma-1) z-\gamma z^{2}}{(1+\gamma)(1-z)^{2}} .
$$

Specializing the parameters $\gamma$ and $n$, we obtain the following operators studied by various authors:

for $f \in A$,

(i) $I_{0}^{n} f(z)=D^{n} f(z)([8])$,

(ii) $I_{\lambda}^{n} f(z)([2],[3],[5])$,

(iii) $I_{1}^{n}=I^{n} f(z)([11])$,

for $f \in H$,

(iv) $I_{0}^{n} f(z)=D^{n} f(z)$ ([7]).

Denote by $S H(\gamma, n, \alpha)$ the subclass of $S H$ consisting of functions $f$ of the form (1) that satisfy the condition

$$
\operatorname{Re}\left(\frac{I_{\gamma}^{n+1} f(z)}{I_{\gamma}^{n} f(z)}\right) \geq \alpha, \quad 0 \leq \alpha<1
$$

where $I_{\gamma}^{n} f(z)$ is defined by (4).

We let the subclass $\overline{S H}(\gamma, n, \alpha)$ consisting of harmonic functions $f_{n}=h+\bar{g}_{n}$ in $S H$ so that $h$ and $g_{n}$ are of the form

$$
h(z)=z-\sum_{k=2}^{\infty} a_{k} z^{k}, g_{n}(z)=(-1)^{n} \sum_{k=1}^{\infty} b_{k} z^{k}, \quad a_{k}, b_{k} \geq 0 .
$$

By suitably specializing the parameters, the classes $S H(\gamma, n, \alpha)$ reduces to the various subclasses of harmonic univalent functions. Such as,

(i) $S H(0,0,0)=S H^{*}(0)$ ( [1], [9], [10]),

(ii) $S H(0,0, \alpha)=S H^{*}(\alpha)$ ( [6]),

(iii) $S H(0,1,0)=K H(0)$ ([1], [9], [10]),

(iv) $\operatorname{SH}(0,1, \alpha)=K H(\alpha)([6])$,

(v) $\operatorname{SH}(0, n, \alpha)=H(n, \alpha)$ ([7]).

Define $S H^{0}(\gamma, n, \alpha):=S H(\gamma, n, \alpha) \cap S H^{0}$ and

$$
\overline{S H}^{0}(\gamma, n, \alpha):=\overline{S H}(\gamma, n, \alpha) \cap S H^{0} \text {. }
$$

\section{Main results}

Theorem 1.Let $f=h+\bar{g}$ be so that $h$ and $g$ are given by (1) with $b_{1}=0$. Furthermore, let

$$
\begin{aligned}
& \sum_{k=2}^{\infty}\left(\frac{k+\gamma}{1+\gamma}\right)^{n}\left(\frac{k+\gamma}{1+\gamma}-\alpha\right)\left|a_{k}\right| \\
& +\sum_{k=2}^{\infty}\left(\frac{k-\gamma}{1+\gamma}\right)^{n}\left(\frac{k-\gamma}{1+\gamma}+\alpha\right)\left|b_{k}\right| \leq 1-\alpha,
\end{aligned}
$$

where $0 \leq \gamma \leq 1 / 2, n \in \mathbb{N}_{0}, \frac{\gamma}{1+\gamma} \leq \alpha \leq \frac{1}{1+\gamma}$. Then $f$ is sense-preserving, harmonic univalent in $U$ and $f \in S H^{0}(\gamma, n, \alpha)$.
Proof.If $z_{1} \neq z_{2}$

$$
\begin{aligned}
\left|\frac{f\left(z_{1}\right)-f\left(z_{2}\right)}{h\left(z_{1}\right)-h\left(z_{2}\right)}\right| & \geq 1-\left|\frac{g\left(z_{1}\right)-g\left(z_{2}\right)}{h\left(z_{1}\right)-h\left(z_{2}\right)}\right| \\
& =1-\left|\frac{\sum_{k=2}^{\infty} b_{k}\left(z_{1}^{k}-z_{2}^{k}\right)}{\left(z_{1}-z_{2}\right)+\sum_{k=2}^{\infty} a_{k}\left(z_{1}^{k}-z_{2}^{k}\right)}\right| \\
& >1-\frac{\sum_{k=2}^{\infty} k\left|b_{k}\right|}{1-\sum_{k=2}^{\infty} k\left|a_{k}\right|} \\
& \geq 1-\frac{\sum_{k=2}^{\infty} \frac{\left(\frac{k-\gamma}{1+\gamma}\right)^{n}\left(\frac{k-\gamma}{1+\gamma}+\alpha\right)}{1-\alpha}\left|b_{k}\right|}{1-\sum_{k=2}^{\infty} \frac{\left(\frac{k+\gamma}{1+\gamma}\right)^{n}\left(\frac{k+\gamma}{1+\gamma}-\alpha\right)}{1-\alpha}\left|a_{k}\right|} \geq 0,
\end{aligned}
$$

which proves univalence. Note that $\mathrm{f}$ is sense preserving in $\mathrm{U}$. This is because

$$
\begin{aligned}
& \left|h^{\prime}(z)\right| \geq 1-\sum_{k=2}^{\infty} k\left|a_{k}\right||z|^{k-1}>1-\sum_{k=2}^{\infty} \frac{\left(\frac{k+\gamma}{1+\gamma}\right)^{n}\left(\frac{k+\gamma}{1+\gamma}-\alpha\right)}{1-\alpha}\left|a_{k}\right| \\
& \geq \sum_{k=2}^{\infty} \frac{\left(\frac{k-\gamma}{1+\gamma}\right)^{n}\left(\frac{k-\gamma}{1+\gamma}+\alpha\right)}{1-\alpha}\left|b_{k}\right|>\sum_{k=2}^{\infty} k\left|b_{k}\right||z|^{k-1} \geq\left|g^{\prime}(z)\right|
\end{aligned}
$$

Using the fact that $R e w \geq \alpha$ if and only if $|1-\alpha+w| \geq|1+\alpha-w|$, it suffices to show that

$$
\left|(1-\alpha) I_{\gamma}^{n} f(z)+I_{\gamma}^{n+1} f(z)\right|-\left|(1+\alpha) I_{\gamma}^{n} f(z)-I_{\gamma}^{n+1} f(z)\right| \geq 0 .
$$

Substituting for $I_{\gamma}^{n} f(z)$ and $I_{\gamma}^{n+1} f(z)$ in (8), we obtain

$$
\begin{array}{r}
\left|(1-\alpha) I_{\gamma}^{n} f(z)+I_{\gamma}^{n+1} f(z)\right|-\left|(1+\alpha) I_{\gamma}^{n} f(z)-I_{\gamma}^{n+1} f(z)\right| \\
\geq 2(1-\alpha)|z|-\sum_{k=2}^{\infty}\left(\frac{k+\gamma}{1+\gamma}\right)^{n}\left(\frac{k+\gamma}{1+\gamma}+1-\alpha\right)\left|a_{k}\right||z|^{k} \\
-\sum_{k=2}^{\infty}\left(\frac{k-\gamma}{1+\gamma}\right)^{n}\left(\frac{k-\gamma}{1+\gamma}-1+\alpha\right)\left|b_{k}\right||z|^{k} \\
-\sum_{k=2}^{\infty}\left(\frac{k+\gamma}{1+\gamma}\right)^{n}\left(\frac{k+\gamma}{1+\gamma}-1-\alpha\right)\left|a_{k}\right||z|^{k} \\
-\sum_{k=2}^{\infty}\left(\frac{k-\gamma}{1+\gamma}\right)^{n}\left(\frac{k-\gamma}{1+\gamma}+1+\alpha\right)\left|b_{k}\right||z|^{k} \\
>2(1-\alpha)|z|\left\{1-\sum_{k=2}^{\infty}\left(\frac{k+\gamma}{1+\gamma}\right)^{n}\left(\frac{k+\gamma}{1+\gamma}-\alpha\right)\left|a_{k}\right|\right. \\
\left.-\sum_{k=2}^{\infty}\left(\frac{k-\gamma}{1+\gamma}\right)^{n}\left(\frac{k-\gamma}{1+\gamma}+\alpha\right)\left|b_{k}\right|\right\} .
\end{array}
$$

This last expression is non-negative by (7), and so the proof is complete. 
Theorem 2.Let $f_{n}=h+\bar{g}_{n}$ be given by (6) with $b_{1}=0$. Then $f_{n} \in \overline{S H}^{0}(\gamma, n, \alpha)$ if and only if

$$
\begin{aligned}
& \sum_{k=2}^{\infty}\left(\frac{k+\gamma}{1+\gamma}\right)^{n}\left(\frac{k+\gamma}{1+\gamma}-\alpha\right) a_{k} \\
& +\sum_{k=2}^{\infty}\left(\frac{k-\gamma}{1+\gamma}\right)^{n}\left(\frac{k-\gamma}{1+\gamma}+\alpha\right) b_{k} \leq 1-\alpha,
\end{aligned}
$$

where $0 \leq \gamma \leq 1 / 2, n \in \mathbb{N}_{0}, \frac{\gamma}{1+\gamma} \leq \alpha \leq \frac{1}{1+\gamma}$.

Proof.The "if" part follows from Theorem 1 upon noting that $\overline{S H}^{0}(\gamma, n, \alpha) \subset S H^{0}(\gamma, n, \alpha)$. For the "only if" part, we show that $f_{n} \notin \overline{S H}^{0}(\gamma, n, \alpha)$ if the condition (9) does not hold. Note that a necessary and sufficient condition for $f_{n}=h+\bar{g}_{n}$ given by (6), to be in $\overline{S H}^{0}(\gamma, n, \alpha)$ is that the condition (5) to be satisfied. This is equivalent to

$$
\begin{gathered}
\operatorname{Re}\left\{\frac{(1-\alpha) z-\sum_{k=2}^{\infty}\left(\frac{k+\gamma}{1+\gamma}\right)^{n}\left(\frac{k+\gamma}{1+\gamma}-\alpha\right) a_{k} z^{k}}{z-\sum_{k=2}^{\infty}\left(\frac{k+\gamma}{1+\gamma}\right)^{n} a_{k} z^{k}+\sum_{k=2}^{\infty}\left(\frac{k-\gamma}{1+\gamma}\right)^{n} b_{k} \bar{z}^{k}}\right. \\
\left.\frac{-\sum_{k=2}^{\infty}\left(\frac{k-\gamma}{1+\gamma}\right)^{n}\left(\frac{k-\gamma}{1+\gamma}+\alpha\right) b_{k} \bar{z}^{k}}{z-\sum_{k=2}^{\infty}\left(\frac{k+\gamma}{1+\gamma}\right)^{n} a_{k} z^{k}+\sum_{k=2}^{\infty}\left(\frac{k-\gamma}{1+\gamma}\right)^{n} b_{k} \bar{z}^{k}}\right\} \geq 0 .
\end{gathered}
$$

The above condition must hold for all values of $z,|z|=r<$ 1. Upon choosing the values of $z$ on the positive real axis where $0 \leq z=r<1$ we must have

$$
\begin{aligned}
& \frac{(1-\alpha)-\sum_{k=2}^{\infty}\left(\frac{k+\gamma}{1+\gamma}\right)^{n}\left(\frac{k+\gamma}{1+\gamma}-\alpha\right) a_{k} r^{k-1}}{1-\sum_{k=2}^{\infty}\left(\frac{k+\gamma}{1+\gamma}\right)^{n} a_{k} r^{k-1}+\sum_{k=2}^{\infty}\left(\frac{k-\gamma}{1+\gamma}\right)^{n} b_{k} r^{k-1}} \\
& \frac{-\sum_{k=2}^{\infty}\left(\frac{k-\gamma}{1+\gamma}\right)^{n}\left(\frac{k-\gamma}{1+\gamma}+\alpha\right) b_{k} r^{k-1}}{1-\sum_{k=2}^{\infty}\left(\frac{k+\gamma}{1+\gamma}\right)^{n} a_{k} r^{k-1}+\sum_{k=2}^{\infty}\left(\frac{k-\gamma}{1+\gamma}\right)^{n} b_{k} r^{k-1}} \geq 0
\end{aligned}
$$

If the condition (9) does not hold, then the numerator in (10) is negative for $r$ sufficiently close to 1 . Hence there exist $z_{0}=r_{0}$ in $(0,1)$ for which the quotient in (10) is negative. This contradicts the required condition for $f_{n} \in$ $\overline{S H}^{0}(\gamma, n, \alpha)$ and so the proof is complete.

Theorem 3.Let $f_{n}$ be given by (6). Then $f_{n} \in \overline{S H}^{0}(\gamma, n, \alpha)$ if and only if

$$
\begin{aligned}
& f_{n}(z)=\sum_{k=1}^{\infty}\left(X_{k} h_{k}(z)+Y_{k} g_{n_{k}}(z)\right) \\
& \text { where } h_{1}(z)=z, \quad g_{n_{1}}(z)=z \\
& h_{k}(z)=z-\frac{1-\alpha}{\left(\frac{k+\gamma}{1+\gamma}\right)^{n}\left(\frac{k+\gamma}{1+\gamma}-\alpha\right)} z^{k}(k=2,3, \ldots), \\
& g_{n_{k}}(z)=z+(-1)^{n} \frac{1-\alpha}{\left(\frac{k-\gamma}{1+\gamma}\right)^{n}\left(\frac{k-\gamma}{1+\gamma}+\alpha\right)} \bar{z}^{k}(k=2,3, \ldots), \\
& \sum_{k=1}^{\infty}\left(X_{k}+Y_{k}\right)=1, X_{k} \geq 0, Y_{k} \geq 0
\end{aligned}
$$

$$
0 \leq \gamma \leq 1 / 2, n \in \mathbb{N}_{0}, \frac{\gamma}{1+\gamma} \leq \alpha \leq \frac{1}{1+\gamma} .
$$

In particular, the extreme points of $\overline{\operatorname{SH}}^{0}(\gamma, n, \alpha)$ are $\left\{h_{k}\right\}$ and $\left\{g_{n_{k}}\right\}$.

Proof.For functions $f_{n}$ of the form (6) we have

$$
\begin{aligned}
f_{n}(z)= & \sum_{k=1}^{\infty}\left(X_{k} h_{k}(z)+Y_{k} g_{n_{k}}(z)\right) \\
= & \sum_{k=1}^{\infty}\left(X_{k}+Y_{k}\right) z-\sum_{k=2}^{\infty} \frac{1-\alpha}{\left(\frac{k+\gamma}{1+\gamma}\right)^{n}\left(\frac{k+\gamma}{1+\gamma}-\alpha\right)} X_{k} z^{k} \\
& +(-1)^{n} \sum_{k=2}^{\infty} \frac{1-\alpha}{\left(\frac{k-\gamma}{1+\gamma}\right)^{n}\left(\frac{k-\gamma}{1+\gamma}+\alpha\right)} Y_{k} \bar{z}^{k} .
\end{aligned}
$$

Then

$$
\begin{aligned}
& \sum_{k=2}^{\infty} \frac{\left(\frac{k+\gamma}{1+\gamma}\right)^{n}\left(\frac{k+\gamma}{1+\gamma}-\alpha\right)}{1-\alpha}\left(\frac{1-\alpha}{\left(\frac{k+\gamma}{1+\gamma}\right)^{n}\left(\frac{k+\gamma}{1+\gamma}-\alpha\right)} X_{k}\right) \\
& +\sum_{k=2}^{\infty} \frac{\left(\frac{k-\gamma}{1+\gamma}\right)^{n}\left(\frac{k-\gamma}{1+\gamma}+\alpha\right)}{1-\alpha}\left(\frac{1-\alpha}{\left(\frac{k-\gamma}{1+\gamma}\right)^{n}\left(\frac{k-\gamma}{1+\gamma}+\alpha\right)} Y_{k}\right) \\
& =\sum_{k=2}^{\infty} X_{k}+\sum_{k=2}^{\infty} Y_{k}=1-X_{1}-Y_{1} \leq 1, \text { and so } f_{n} \in \overline{S H}^{0}(\gamma, n, \alpha) .
\end{aligned}
$$

Conversely, if $f_{n} \in \overline{S H}^{0}(\gamma, n, \alpha)$, then

$$
a_{k} \leq \frac{1-\alpha}{\left(\frac{k+\gamma}{1+\gamma}\right)^{n}\left(\frac{k+\gamma}{1+\gamma}-\alpha\right)}
$$

and

$$
b_{k} \leq \frac{1-\alpha}{\left(\frac{k-\gamma}{1+\gamma}\right)^{n}\left(\frac{k-\gamma}{1+\gamma}+\alpha\right)}
$$

Set

$$
\begin{aligned}
& X_{k}=\frac{\left(\frac{k+\gamma}{1+\gamma}\right)^{n}\left(\frac{k+\gamma}{1+\gamma}-\alpha\right)}{1-\alpha} a_{k},(k=2,3, \ldots) \\
& Y_{k}=\frac{\left(\frac{k-\gamma}{1+\gamma}\right)^{n}\left(\frac{k-\gamma}{1+\gamma}+\alpha\right)}{1-\alpha} b_{k},(k=2,3, \ldots)
\end{aligned}
$$

and

$$
X_{1}+Y_{1}=1-\left(\sum_{k=2}^{\infty} X_{k}+Y_{k}\right)
$$

where $X_{k}, Y_{k} \geq 0$. Then, as required, we obtain

$f_{n}(z)=\left(X_{1}+Y_{1}\right) z+\sum_{k=2}^{\infty} X_{k} h_{k}(z)+\sum_{k=2}^{\infty} Y_{k} g_{n_{k}}(z)=\sum_{k=1}^{\infty}\left(X_{k} h_{k}(z)+Y_{k} g_{n_{k}}(z)\right)$.

Theorem 4.Let $f_{n} \in \overline{S H}^{0}(\gamma, n, \alpha)$. Then for $|z|=r<1$ and $0 \leq \gamma \leq 1 / 2, n \in \mathbb{N}_{0}, \frac{\gamma}{1+\gamma} \leq \alpha \leq \frac{1}{1+\gamma}$ we have 


$$
\left|f_{n}(z)\right| \leq r+\frac{(1-\alpha)}{\left(\frac{2+\gamma}{1+\gamma}\right)^{n}\left(\frac{2+\gamma}{1+\gamma}-\alpha\right)} r^{2}
$$

and

$$
\left|f_{n}(z)\right| \geq r-\frac{(1-\alpha)}{\left(\frac{2+\gamma}{1+\gamma}\right)^{n}\left(\frac{2+\gamma}{1+\gamma}-\alpha\right)} r^{2} .
$$

Proof.We only prove the right hand inequality. The proof for the left hand inequality is similar and will be omitted. Let $f_{n} \in \overline{S H}^{0}(\gamma, n, \alpha)$. Taking the absolute value of $f_{n}$ we have

$$
\begin{array}{r}
\left|f_{n}(z)\right| \leq r+\sum_{k=2}^{\infty}\left(a_{k}+b_{k}\right) r^{2} \\
\leq r+\frac{(1-\alpha) r^{2}}{\left(\frac{2+\gamma}{1+\gamma}\right)^{n}\left(\frac{2+\gamma}{1+\gamma}-\alpha\right)} \\
\times \sum_{k=2}^{\infty}\left\{\frac{\left(\frac{k+\gamma}{1+\gamma}\right)^{n}\left(\frac{k+\gamma}{1+\gamma}-\alpha\right)}{1-\alpha} a_{k}+\frac{\left(\frac{k-\gamma}{1+\gamma}\right)^{n}\left(\frac{k-\gamma}{1+\gamma}+\alpha\right)}{1-\alpha} b_{k}\right\} \\
\leq r+\frac{(1-\alpha)}{\left(\frac{2+\gamma}{1+\gamma}\right)^{n}\left(\frac{2+\gamma}{1+\gamma}-\alpha\right)} r^{2} .
\end{array}
$$

The following covering result follows from the left hand inequality in Theorem 4.

Corollary 1.Let $f_{n}$ of the form (6) be so that $f_{n} \in \overline{S H}^{0}(\gamma, n, \alpha)$, where $0 \leq \gamma \leq 1 / 2, n \in \mathbb{N}_{0}$, $\frac{\gamma}{1+\gamma} \leq \alpha \leq \frac{1}{1+\gamma}$. Then

$$
\left\{w:|w|<1-\frac{(1-\alpha)}{\left(\frac{2+\gamma}{1+\gamma}\right)^{n}\left(\frac{2+\gamma}{1+\gamma}-\alpha\right)}\right\} \subset f_{n}(U) .
$$

Theorem 5.The class $\overline{\mathrm{SH}}^{0}(\gamma, n, \alpha)$ is closed under convex combinations.

Proof.Let $f_{n_{i}} \in \overline{S H}^{0}(\gamma, n, \alpha)$ for $i=1,2, \ldots$, where $f_{n_{i}}$ is given by

$$
f_{n_{i}}(z)=z-\sum_{k=2}^{\infty} a_{k_{i}} z^{k}+(-1)^{n} \sum_{k=2}^{\infty} b_{k_{i}} \bar{z}^{k} .
$$

Then by (9),

$$
\sum_{k=2}^{\infty} \frac{\left(\frac{k+\gamma}{1+\gamma}\right)^{n}\left(\frac{k+\gamma}{1+\gamma}-\alpha\right)}{1-\alpha} a_{k_{i}}+\sum_{k=2}^{\infty} \frac{\left(\frac{k-\gamma}{1+\gamma}\right)^{n}\left(\frac{k-\gamma}{1+\gamma}+\alpha\right)}{1-\alpha} b_{k_{i}} \leq 1 .
$$

For $\sum_{i=1}^{\infty} t_{i}=1,0 \leq t_{i} \leq 1$, the convex combination of $f_{n_{i}}$ may be written as

$\sum_{i=1}^{\infty} t_{i} f_{n_{i}}(z)=z-\sum_{k=2}^{\infty}\left(\sum_{i=1}^{\infty} t_{i} a_{k_{i}}\right) z^{k}+(-1)^{n} \sum_{k=2}^{\infty}\left(\sum_{i=1}^{\infty} t_{i} b_{k_{i}}\right) \bar{z}^{k}$.
Then by (11),

$$
\begin{aligned}
& \sum_{k=2}^{\infty} \frac{\left(\frac{k+\gamma}{1+\gamma}\right)^{n}\left(\frac{k+\gamma}{1+\gamma}-\alpha\right)}{1-\alpha}\left(\sum_{i=1}^{\infty} t_{i} a_{k_{i}}\right) \\
+ & \sum_{k=2}^{\infty} \frac{\left(\frac{k-\gamma}{1+\gamma}\right)^{n}\left(\frac{k-\gamma}{1+\gamma}+\alpha\right)}{1-\alpha}\left(\sum_{i=1}^{\infty} t_{i} b_{k_{i}}\right) \\
= & \sum_{i=1}^{\infty} t_{i}\left\{\sum_{k=2}^{\infty} \frac{\left(\frac{k+\gamma}{1+\gamma}\right)^{n}\left(\frac{k+\gamma}{1+\gamma}-\alpha\right)}{1-\alpha} a_{k_{i}}+\frac{\left(\frac{k-\gamma}{1+\gamma}\right)^{n}\left(\frac{k-\gamma}{1+\gamma}+\alpha\right)}{1-\alpha} b_{k_{i}}\right\}
\end{aligned}
$$

$\leq \sum_{i=1}^{\infty} t_{i}=1$

This is the condition required by (9) and so $\sum_{i=1}^{\infty} t_{i} f_{n_{i}}(z) \in \overline{S H}^{0}(\gamma, n, \alpha)$.

\section{References}

[1] Y. Avc1, E. Zlotkiewicz, Ann. Univ. Mariae CurieSklodowska Sect. A., 44, 1-7 (1990).

[2] N. E. Cho, H. M. Srivastava, Math. Comput. Modelling, 37, 39-49 (2003).

[3] N. E. Cho, T. H. Kim, Bull. Korean Math. Soc., 40, 399-410 (2003).

[4] J. Clunie, T. Sheil-Small, Ann. Acad. Sci. Fenn. Ser. A I Math., 9, 3-25 (1984).

[5] T. M. Flett, J. Math. Anal. Appl., 38, 746-765 (1972).

[6] J. M. Jahangiri, J. Math. Anal. Appl., 235, 470-477 (1999).

[7] J. M. Jahangiri, G. Murugusundaramoorthy, K. Vijaya, South J. Pure Appl. Math., 2, 77-82, (2002).

[8] G. S. Salagean, Lecture Notes in Math. Springer- Verlag Heidelberg 1013, 362-372 (1983).

[9] H. Silverman, J. Math. Anal. Appl., 220, 283-289 (1998).

[10] H. Silverman, E. M. Silvia, N. Z. J. Math. 28, 275-284 (1999).

[11] B. A. Uralegaddi and C. Somanatha, Current topics in analytic function theory, In: H. M. Srivastava and S. Owa (Eds.), World Sci. Publishing, Singapore, 371-374 (1992). 


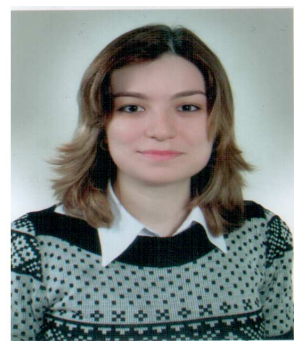

Elif Yaşar received her M.Sc. and Ph.D. degrees in Mathematics from the Uludag University of Bursa, in 2007 and 2012, respectively. Her research interests are in the geometric function theory. Especially, harmonic mappings, convolution and hypergeometric functions.

\section{Sibel Yalçın}

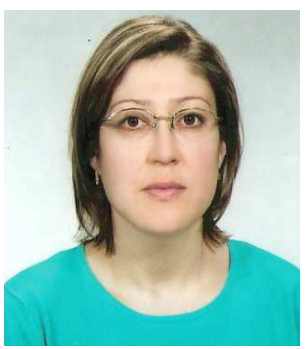

received her $\mathrm{Ph} . \mathrm{D}$. degree in Mathematics in 2001 from the Uludag University of Bursa, Turkey. She became a full Professor in 2011. She is currently with the Department of Mathematics, Uludag University. Her research interests include harmonic mappings, geometric function theory, meromorphic functions, convolution operators. She has published research articles in reputed international journals of mathematics. 